\title{
EDITORIAL
}

\section{The evolution of trial registration}

\author{
With mandatory clinical trial registration now in place for most novel therapeutic \\ interventions, key questions remain on how to maximize the potential benefits of the \\ enhanced transparency that this provides.
}

Five years ago, the International Committee of Medical Journal Editors (ICMJE) published an editorial stating that, from mid 2005, its member journals would only consider papers reporting trial results for publication if the trial had been registered at its outset in a publicly accessible registry ${ }^{1}$. The key aim of the policy was to promote registration of clinical trials, which facilitates patient enrolment and could also help address the problem of 'publication bias' - selective reporting of positive results - by allowing all data associated with a given therapeutic intervention to be assessed.

At the time, the only clinical trial registry that fulfilled all of the criteria set out by the ICMJE was ClinicalTrials.gov, which was established by the US National Library of Medicine in 2000 to provide the public with access to information about ongoing clinical trials in which they might be able to participate. Following the announcement of the ICMJE policy, the number of trials registered at ClinicalTrials.gov increased dramatically, and it now contains data on almost 80,000 trials.

The importance of this registry is also increasing owing to the FDA Amendments Act of 2007, which requires that trials beyond the Phase I stage involving products that are subject to FDA regulation must be registered at ClinicalTrials.gov. Furthermore, it is now required that the key characteristics and results of the trial are also disclosed in the registry, generally within 1 year of trial completion.

Given this, it is of interest to understand how the nature of the data at ClinicalTrials.gov is evolving, and two recent studies provide some insight into this issue $e^{2,3}$. The first of these investigated the registration characteristics of trials that were published in high-impact journals in 2008 in three medical areas: cardiology, rheumatology and gastroenterology. The authors found that, of the 323 trials analysed, $147(\sim 46 \%)$ were adequately registered (that is, before the end of the trial and with a clearly specified primary outcome). Of these, $31 \%$ of the associated articles showed some evidence of discrepancies between the registered and reported outcomes, raising concerns about selective outcome reporting ${ }^{2}$.

The second study took an alternative approach, and examined the publication rates and registration data for trials registered at ClinicalTrials.gov by using a random 10\% subsample of trials registered after 31 December 1999 and completed before 1 January 2006, to allow at least 2 years for publication following completion. Among this sample, fewer than half ( 311 of $677 ; 46 \%$ ) were published. The rate of publication was $40 \%$ for the 357 trials sponsored primarily by industry, $47 \%$ for 122 governmentsponsored trials (a difference that was not statistically significant) and 56\% for the other 198 trials $^{3}$.

Although initial consideration of the two studies might suggest that clinical trial registration is not yet addressing publication bias, they indicate that registration is providing the opportunity to identify relevant deficiencies. Furthermore, the limitations of the studies might make it more appropriate to consider them as a useful benchmark in the future to gauge the impact of the ICMJE policy and the recent changes to US legislation. For example, in the first study, of the total 234 registered trials that were analysed, 195 (83\%) had enrolled participants before the ICJME policy came into effect in 2005. Similarly, only a small proportion of the trials analysed in the second study were in progress after the policy introduction.

The two studies also raise important questions for those involved in the process of publishing trial results. For example, the first study suggests that journal editors and peer reviewers did not routinely use opportunities to check differences between the outcomes that were listed for registered trials at ClinicalTrials.gov and those in the papers reporting these trials. The second study highlights the long-standing issue of how best to disseminate the results of negative and inconclusive trials. These may well represent the bulk of the unpublished trials identified in the study and, without greater efforts by journals to support the publication of such trials, it seems unlikely that the proportion of these that are published will change rapidly. Third, the recent legal requirement for prompt disclosure of trial results to an extent that would traditionally preclude subsequent publication in leading medical journals raises a novel question for such journals over their criteria in this respect. Given these issues, it is clear that debate and adaptation among the key stakeholders in clinical research is now needed to maximize the benefits offered by the greater transparency in clinical trial reporting.

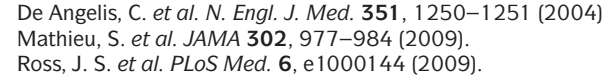

